\title{
Continuous Motion Planning for Information Forecast
}

\author{
Han-Lim Choi and Jonathan P. How
}

\begin{abstract}
This paper addresses planning of continuous paths for mobile sensors to improve long-term forecast performance. With the information gain defined by the mutual information between the continuous measurement path and the future verification variables, two expressions for computing the information gain for a linear time-varying system are derived: the filter form and the smoother form. The smoother form, inspired by the conditional independence structure, is shown to be preferable, since it does not require integration of differential equations for long time intervals, it simplifies the process of calculating the accumulated information on the fly, and its time derivative extracts out the pure impact of sensing regardless of the process noise. Utilizing the spatial interpolation technique to relate the sensor movement to the evolution of the observation matrix, the optimal path planning formulation and the real-time steering law are presented. A numerical example of a simplified weather forecast validates the proposed methodology.
\end{abstract}

\section{INTRODUCTION}

Motion planning of robotic sensors to gather information has been recently spotlighted in the context of tracking moving targets [1]-[4]. The mutual information has often been employed as the information metric, because it represents the uncertainty reduction of the quantity of interest by sensor measurements. [1] presented sensor coordination based on maximization of the average mutual information between the measurement and the target state, while [2] suggested a particle filter-based distributed control method, which optimizes the mutual information between the measurement and the instantaneous state.

This work considers motion planning of sensors that measure the environmental field variables (e.g. UAVs equipped with pressure and temperature sensors) with the information metric of mutual information between the measurement and a subset of the future state. This motion planning is related to the previous works on path planning for target tracking, as it shares the idea of representation of vehicle motion as changes in the observation function. However, there are two noticeable aspects in this work: first, fully continuous aspects of the vehicle motion in both time and space manner are considered, and second, the interest is in long-term forecast for a specified verification variables rather than tracking of the target state within short-term planning window.

The spatially continuous feature of the vehicle path can be addressed by using spatial interpolation techniques such as Kriging [5] and Gaussian Processes Regression [6] that predict a value of the quantity of interest at arbitrary points in continuous space as a function of values at a finite number of grid points. Using this technique, this work relates

H. -L. Choi and J. P. How are with the Dept. of Aeronautics and Astronautics, MIT, Cambridge, MA 02139, USA, \{hanlimc, jhow\} @mit.edu measurement action in a continuous spatial domain to an observation function, which is similar to previous path planning literature. However, the temporally continuous aspects of the measurement paths and their relation to future forecasts have not been extensively studied in the path planning context. [7] addressed continuous path planning based on some type of information rate, but it did not explicitly quantify the mutual information. This paper presents an explicit way of computing the mutual information between the continuous measurement path and the future verification variables.

In the context of information theory, there has been a long history of research about the mutual information between the signal and observation in a continuous-time domain. Duncan [8] showed that the mutual information between the signal history and observation history (i.e. signal during $[0, t]$ and observation during $[0, t])$ is expressed as a function of estimation error for a Gaussian signal through an additive Gaussian channel. Similar quantification is performed for non-Gaussian signal $[9,10]$ and fractional Gaussian channel [11]. On the other hand, [12] showed that the optimal filter for a linear system that maximizes the mutual information between the observation history for $[0, t]$ and the state value at $t$ is Kalman-Bucy filter; [13] related this mutual information to the Fisher information matrix. Recently, Mitter and Newton [14] presented an expression for the mutual information between the signal path during $[s, t], s<t$ and the observation history, with statistical mechanical interpretation of this expression. Newton $[15,16]$ extended his previous results by quantifying the mutual information between the future signal path during $[t, T], t \leq$ $T$ and the past measurement history during $[0, t]$ for linear time-varying [15] and nonlinear [16] systems. However, it should be noted that all these previous quantifications have been about the state and observation. In contrast, this work deals with the mutual information between the values of a subset of the state at $T$ and the observation history during $[0, t]$ when $T>t$.

Regarding the quantification of the mutual information, this work first presents the filter form, which is a simple extension of the previous work [12]-[14], by treating the forecast problem as a filtering problem with longer time window. However, it then shows that this form might not be suitable for motion planning for a long-term forecast in three senses: sensitivity to the model accuracy, computational cost, and the lack of on-the-fly knowledge of the accumulated information. Alternatively, the smoother form is suggested, which regards forecasting as fixed-interval smoothing. The equivalence of the smoother form to the filter form is proved based on the conditional independence of the measurement 

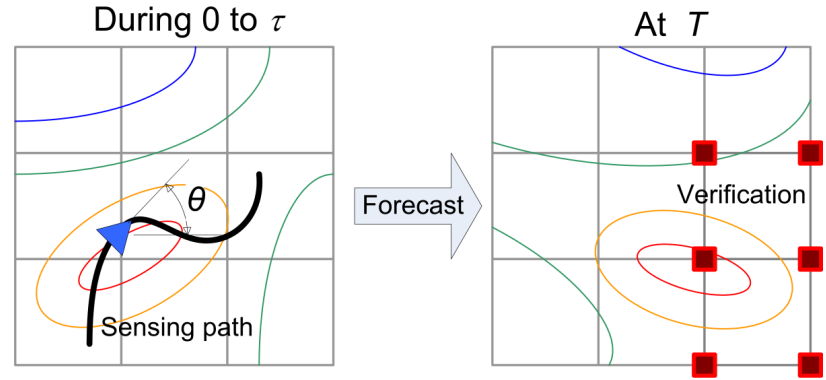

Fig. 1. Continuous motion planning of a sensor for best information forecast: a mobile sensor senses some environmental variable shown in contour along the path designed to achieve best forecast for the verification region.

history and the future verification variables for a given present state value. Using the covariance equations for a twofilter type smoothing in [17], this work derives the expression for the mutual information in the forecast problem involving matrix differential equations that are only integrated over the planning time window. These features resolve all of the key problems identified with the filter formulation.

This work presents a general methodology of continuous motion planning for forecast problems by combining the smoother form quantification of the information associated with a temporally continuous measurement history, and the spatial interpolation-based representation of spatially continuous measurement. In addition to an optimal path planning formulation that maximizes the mutual information, a realtime steering law based on the extension of the information potential field [7] is presented. A numerical example with a linearized Lorenz-2003 chaos system validates the suggested methodological framework.

\section{PROBlem Description}

This paper addresses continuous motion planning for mobile sensors to improve forecast over the specified region. In Fig. 1, a mobile sensor continuously takes measurements of environmental field variables along a continuous path during the time interval $[0, \tau]$, while the quantity of interest is the forecast performance over the verification region, which is a sub-region of the entire world, at the verification time $T$. The objective of the motion planning in this work is to design a path for the sensor (or paths for the multiple sensors) that leads to the largest uncertainty reduction in the forecast, which is represented by the notion of mutual information. The weather forecasting is a motivating application of this planning decision, the goal being design of an adaptive sensor network that supplements fixed observation networks for the sake of improvement of the forecast for a specific region [18]-[21].

There are two main aspects in this problem: 1) quantification of the information gain associated with a continuous (in both the temporal and spatial sense) measurement, and 2) path planning techniques that provide optimal (or good suboptimal) solutions. This paper will define the information gain for temporally continuous measurement and will suggest formula for effective quantification of the information gain. Then, a finite dimensional representation of a spatially continuous path will be provided. This paper will formulate an optimal motion planning problem and also present a realtime motion planning law.

\section{INFORMATION BY CONTINUOUS MEASUREMENT}

Consider an environmental dynamics of state variables $X_{t} \in \mathbb{R}^{n}$ that is described by the following linear timevarying system:

$$
\dot{X}_{t}=A(t) X_{t}+W_{t}
$$

where $W_{t} \in \mathbb{R}^{n}$ is a zero-mean Gaussian noise with $\mathbb{E}\left[W_{t} W_{s}^{\prime}\right]=\Sigma_{W} \delta(t-s), \quad \Sigma_{W} \succeq 0$, which is independent of $X_{t}$. The prime sign' denotes the transpose of a matrix. The initial condition of the state, $X_{0}$ is normally distributed as $X_{0} \sim \mathcal{N}\left(\mu_{0}, P_{0}\right), P_{0} \succ 0$. This work considers a linear measurement model for $Z_{t} \in \mathbb{R}^{m}$ with additive Gaussian noise:

$$
Z_{t}=C(t) X_{t}+N_{t}
$$

where $N_{t} \in \mathbb{R}^{m}$ is zero-mean Gaussian with $\mathbb{E}\left[N_{t} N_{s}^{\prime}\right]=$ $\Sigma_{N} \delta(t-s), \quad \Sigma_{N} \succ 0$, which is independent of $X_{t}$ and $W_{s}, \forall s$. A linear sensing model can be a good representation of observation of environmental variables distributed in field, such as temperature and pressure.

With this environmental system model, this work is interested in determining the impact of a measurement history in the past on the uncertainty reduction of some verification variables in the future. A measurement history up to time $t$ is defined as

$$
\mathcal{Z}_{t}=\left\{Z_{s}: s \in[0, t]\right\}
$$

The verification variables are a subset of the state variables that can be expressed as

$$
V_{t}=M_{V} X_{t} \in \mathbb{R}^{p}
$$

where $M_{V} \in\{0,1\}^{p \times n}, \quad p<n$ with every row-sum of $M_{V}$ being unity. Although this work is specifically interested in the case entries of $M_{V}$ are zero or one, the results can be easily extended to a general $M_{V} \in \mathbb{R}^{p \times n}$.

Employing entropy as a metric of uncertainty, the uncertainty reduction of a random quantity by another random quantity is expressed as the mutual information between them. The information gain by a measurement path, in this work, is defined as follows:

$$
\mathcal{J}_{V}(T, \tau) \triangleq \mathcal{I}\left(V_{T} ; \mathcal{Z}_{\tau}\right), \quad 0 \leq \tau<T,
$$

which is the mutual information between $V_{T}$ and $\mathcal{Z}_{\tau}$. This represents the entropy reduction of verification variables in the future time $T$ by the measurement history up to time $\tau$, and also the entropy reduction of $\mathcal{Z}_{\tau}$ by $V_{T}$.

\section{A. Filter Form}

For linear Gaussian systems, there are known expressions for the mutual information between the state variables at a given time and the measurement history up to that time [12][14]. Therefore, one way to compute the information gain is to consider a filtering problem that estimates $X_{T}$ based on the measurement history up to time $T$ denoted as $\mathcal{Z}_{T} \triangleq$ 
$\mathcal{Z}_{\tau} \cup \emptyset_{[\tau, T]}$ where $\emptyset_{[\tau, T]}$ means that no measurement is taken during $[\tau, T]$. Then, $\mathcal{I}\left(X_{T} ; \mathcal{Z}_{T}\right)=\mathcal{I}\left(X_{T} ; \mathcal{Z}_{\tau}\right)$, because no information is gathered by an empty measurement. This procedure of obtaining $\mathcal{I}\left(X_{T} ; \mathcal{Z}_{\tau}\right)$ can be extended when our interest is in $\mathcal{I}\left(V_{T} ; \mathcal{Z}_{\tau}\right)$, as outlined in the following proposition:

Proposition 1 (Filter Form) For the linear system described in this paper, the information gain can be computed as

$$
\begin{aligned}
& \mathcal{J}_{V}^{F}(T, \tau) \triangleq \mathcal{I}\left(V_{T} ; \mathcal{Z}_{\tau}, \emptyset_{[\tau, T]}\right) \\
& \quad=\frac{1}{2} \operatorname{ldet}\left(M_{V} P_{X}(T) M_{V}^{\prime}\right)-\frac{1}{2} \operatorname{ldet}\left(M_{V} Q_{X}(T) M_{V}^{\prime}\right)
\end{aligned}
$$

where $\operatorname{ldet}(\cdot)$ stands for $\log \operatorname{det}(\cdot)$ of a symmetric positive definite matrix, and the superscript ' $F$ ' stands for the filter form. $P_{X}(T)$ and $Q_{X}(T)$ are obtained by integrating the following matrix differential equations:

$$
\begin{aligned}
\dot{P}_{X}(t)= & A(t) P_{X}(t)+P_{X}(t) A^{\prime}(t)+\Sigma_{W} \\
\dot{Q}_{X}(t)= & A(t) Q_{X}(t)+Q_{X}(t) A^{\prime}(t) \\
& +\Sigma_{W}-\mathbb{I}_{[0, \tau]}(t) Q_{X}(t) C(t)^{\prime} \Sigma_{N}^{-1} C(t) Q_{X}(t)
\end{aligned}
$$

with initial conditions $P_{X}(0)=Q_{X}(0)=P_{0} \succ 0$, and $\mathbb{I}_{[0, \tau]}(t): \mathbb{R}_{+} \mapsto\{0,1\}$ is the indicator function that is unity for $t \in[0, \tau]$ and zero elsewhere. The above equations are well-defined for finite $T$ with $P_{0} \succ 0$.

Proof: The key ideas of the proof are that $\mathcal{I}\left(V_{T} ; \mathcal{Z}_{\tau}\right)=$ $\mathcal{I}\left(V_{T} ; \widehat{V}_{T}\right)$ where $\widehat{V}_{T}$ is the Bayesian estimate of $V_{T}$ given $\mathcal{Z}_{\tau}$, and that the remaining uncertainty of $V_{T}$ given $\widehat{V}_{T}$ is the estimation error $V_{T}-\widehat{V}_{T}$ whose covariance can be obtained from (9). See Proposition 3 in [22, Chapter 5] for details.

The filter form provides a simple expression for the information gain for the future verification variables by a continuous past measurement history, which is described in terms of the solution of a Lyapunov equation and a Riccati equation. However, this form of computation involves the following issues, particularly, in case the forecast horizon is much longer than the planning horizon, i.e. $T \gg \tau$ :

\section{Remark 1 (Issues in filter form)}

(a) In many engineering problems, a linear model is used for the representation of the short time-scale dynamic behavior of a nonlinear system. Thus, the filter form of the information gain is valid in case the quality of the linear model is guaranteed for the time horizon $[0, T]$. If $T$ is large, this might not be true.

(b) The computational complexity of numerical integration is linear in the integration time interval; thus, the computation time for computing $\mathcal{J}_{V}^{F}(T, \tau)$ linearly increases with respect to $T$. This is not a problem when one is interested in computation of the information gain for few measurement histories. However, if the goal is to design an optimal measurement path, and the computation of information gains for many different candidate measurement histories is needed, long integration can cause computational inefficiency.

(c) The on-the-fly knowledge about the information achieved by the measurement taken thus far can be useful for realtime path planning decision. However, in the filter form expression, this information requires significant amount of further computation. For instance, in order to compute the information attained by measurement history $\mathcal{Z}_{\sigma}, \sigma<\tau$ at time $\sigma$, further integration of $Q_{X}(t)$ over $[\sigma, T]$ should be conducted.

\section{B. Smoother Form}

This section will propose a smoother form for the quantification of the information gain, which resolves all the issues in the filter form listed in Remark 1. It can be shown that the differential equations for the smoother form are essentially integrated for the planning time horizon, and that the information attained by the measurement thus far can be immediately computed without significant computation. This section first suggests an alternative expression of the mutual information that applies to any nonlinear non-gaussian cases:

Proposition 2 If state dynamics satisfy the Markov property, i.e. a future state is conditionally independent of a past state given the present state, and the measurement noise is independent of future process noise, then

$$
\mathcal{I}\left(V_{T} ; \mathcal{Z}_{\tau}\right)=\mathcal{I}\left(X_{\tau} ; \mathcal{Z}_{\tau}\right)-\mathcal{I}\left(X_{\tau} ; \mathcal{Z}_{\tau} \mid V_{T}\right)
$$

In other words, $\mathcal{I}\left(V_{T} ; \mathcal{Z}_{\tau}\right)$ can be interpreted as the difference between the information about $X_{\tau}$ contained in $\mathcal{Z}_{\tau}$, before and after $V_{T}$ is revealed.

Proof: The key proof idea is $\mathcal{I}\left(V_{T} ; \mathcal{Z}_{\tau} \mid X_{\tau}\right)=0$, since $V_{T}$ and $\mathcal{Z}_{\tau}$ are conditionally independent each other given $X_{\tau}$. See Proposition 4 in [22, Chapter 5] for details.

For linear systems with Gaussian noise described by (1) and (2), the mutual information between $X_{\tau}$ and $\mathcal{Z}_{\tau}$ can be easily computed by using a known expression in the literature [12]-[14]. The conditional mutual information $\mathcal{I}\left(X_{\tau} ; \mathcal{Z}_{\tau} \mid V_{T}\right)$ can be quantified by posing a fixed-interval smoothing problem that incorporates the continuous measurement history $\mathcal{Z}_{\tau}$ and the discrete noise-free measurement of verification variables at time $T$ :

Proposition 3 (Smoother Form) If $P_{0 \mid V} \triangleq \operatorname{Cov}\left(X_{0} \mid V_{T}\right) \succ$ 0 is available, the information gain $\mathcal{J}_{V}(T, \tau)$ can be computed as

$$
\begin{aligned}
\mathcal{J}_{V}^{S}(T, \tau) & \triangleq \mathcal{I}\left(X_{\tau} ; \mathcal{Z}_{\tau}\right)-\mathcal{I}\left(X_{\tau} ; \mathcal{Z}_{\tau} \mid V_{T}\right) \\
& =\mathcal{J}_{0}(\tau)-\frac{1}{2} \operatorname{ldet}\left(I+Q_{X}(\tau) \Delta_{S}(\tau)\right)
\end{aligned}
$$

where $\mathcal{J}_{0} \triangleq \frac{1}{2} l \operatorname{det} S_{X \mid V}-\frac{1}{2} \operatorname{ldet} S_{X}, \Delta_{S} \triangleq S_{X \mid V}-S_{X}$, and $S_{X}, S_{X \mid V}$, and $Q_{X}$ are determined by the following matrix differential equations:

$$
\begin{aligned}
\dot{S}_{X}= & -S_{X} A-A^{\prime} S_{X}-S_{X} \Sigma_{W} S_{X} \\
\dot{S}_{X \mid V}= & -S_{X \mid V}\left(A+\Sigma_{W} S_{X}\right)-\left(A+\Sigma_{W} S_{X}\right)^{\prime} S_{X \mid V} \\
& +S_{X \mid V} \Sigma_{W} S_{X \mid V} \\
\dot{Q}_{X}= & A Q_{X}+Q_{X} A^{\prime}+\Sigma_{W}-Q_{X} C^{\prime} \Sigma_{N}^{-1} C Q_{X}
\end{aligned}
$$

with initial conditions $S_{X}(0)=P_{0}^{-1}, S_{X \mid V}(0)=P_{0 \mid V}^{-1}$, and $Q_{X}(0)=P_{0}$. The superscript ' $S$ ' denotes the smoother form, and the time argument is omitted as no confusion is 
expected.

Proof: The sketchy proof is as follows. For a linear Gaussian setting, (10) is stated as

$$
\begin{aligned}
\mathcal{I}\left(V_{T} ; \mathcal{Z}_{\tau}\right)=\frac{1}{2}[ & \left.-\operatorname{ldet} S_{X}(\tau)-\operatorname{ldet} Q_{X}(\tau)\right] \\
& -\frac{1}{2}\left[-\operatorname{ldet} S_{X \mid V}(\tau)-\operatorname{ldet} Q_{X \mid V}(\tau)\right]
\end{aligned}
$$

where $S_{X}(\tau) \triangleq \operatorname{Cov}\left(X_{\tau} \mid \mathcal{Z}_{\tau}\right)^{-1}, S_{X \mid V}(\tau) \triangleq \operatorname{Cov}\left(X_{\tau} \mid V_{T}\right)^{-1}$, $Q_{X}(\tau) \triangleq \operatorname{Cov}\left(X_{\tau} \mid \mathcal{Z}_{\tau}\right)$, and $Q_{X \mid V}(\tau) \triangleq \operatorname{Cov}\left(X_{\tau} \mid \mathcal{Z}_{\tau}, V_{T}\right)$. It was shown in [17] that $Q_{X \mid T}^{-1}=Q_{X}^{-1}+S_{X \mid V}-S_{X}$; therefore, $\operatorname{ldet} Q_{X}-\operatorname{ldet} Q_{X \mid T}=\operatorname{ldet}\left(I+Q_{X} \Delta_{S}\right)$. See Proposition 5 in [22, Chapter 5] for details.

For the linear setting as in this work, $P_{0 \mid V}$ can be computed by the covariance update formula:

$P_{0 \mid V}=P_{0}-P_{0} \Phi_{(T, 0)}^{\prime} M_{V}^{\prime}\left[M_{V} P_{X}(T) M_{V}^{\prime}\right]^{-1} M_{V} \Phi_{(T, 0)} P_{0}$.

where $\Phi_{(T, 0)}$ is the state transition matrix from time 0 to $T$. Note that the inverse on the right-hand side exists for finite $T$ with $P_{0} \succ 0$. A fixed-point smoothing using state augmentation can be easily applied for finding $P_{0 \mid V}$. In case the linear system is used to approximate short-term behavior of a nonlinear system whose long-term behavior is tracked by some nonlinear estimation scheme, $P_{0 \mid V}$ information can be provided by this nonlinear estimator. For instance, in the ensemble-based estimation framework, the ensemble augmentation technique presented by the authors [21] can be used for this purpose.

Remark 2 (Resolution of Issues in Filter Form) Note that given $P_{0 \mid V}$, every differential equation for the smoother form is integrated forward up to time $\tau$. The only part for which a long integration might be involved in is the calculation of the conditional initial covariance $P_{0 \mid V}$, which is performed only once before incorporating the measurement history.

(a) Regarding the model validity issue in Remark 1, if $P_{0 \mid V}$ is provided from a nonlinear estimation scheme, the time interval over which the validity of the linear model needs to be guaranteed is $[0, \tau]$, as the long-term nonlinear feature of dynamics is incorporated in $P_{0 \mid V}$. Even when there is no such nonlinear estimator, the smoother form allows for pinpointing the possible effect of the limited model validity to the sole term $P_{0 \mid V}$, which enables separate analysis of improving the quality of this term.

(b) As to computational complexity, only one quantity, $Q_{X}(\tau)$ is dependent on the measurement history. Thus, if one needs to evaluate the information gains for different measurement histories, then one can simply integrate the Ricatti equation to get $Q_{X}(\tau)$, while $\mathcal{J}_{0}$ and $\Delta_{S}$ have been computed in advance.

(c) For the smoother form, the information gain accomplished by a partial measurement history $\mathcal{Z}_{\sigma}, \sigma<\tau$ is straightforwardly computed as $\mathcal{J}_{V}^{S}(T, \sigma)$, the same expression as (12) with the matrix values evaluated for time $\sigma$. Since all the matrix differential equations are integrated forward, $\mathcal{J}_{V}^{S}(T, \sigma)$ is available in real time without further computation. The on-the-fly information will be discussed in detail in section III-C with the notion of mutual information rate.

Conventional path planning problems [7] aim for reducing the uncertainty in the state estimate at the end of the planning window: i.e. min ldet $Q_{X}(\tau)$. Recalling that the forecast problem written in the smoother form considers min ldet $\left(I+Q_{X}(\tau) \Delta_{S}(\tau)\right)$, the smoother form objective function can be regarded as some weighted version of the conventional objective function. Depending on $\Delta_{S}(\tau)$, the solutions of the two problems can be very different; however, a planning algorithm for the conventional decision can be easily extended to the forecast problem, since the smoother enables projection of the decision space from a long forecast horizon $[0, T]$ on to a short planning horizon $[0, \tau]$.

\section{Mutual Information Rate and On-The-Fly Information}

This section analyzes time derivative of the mutual information and the on-the-fly accumulated information to identify important features of the smoother form expression in terms of information supply and dissipation.

While computing the filter form mutual information $\mathcal{J}_{V}^{F}(T, \tau)$, the following two quantities are available on the fly at time $t<\tau$ : the mutual information between the current state and the measurement thus far, $\mathcal{I}\left(X_{t} ; \mathcal{Z}_{t}\right)$, and its projection on to the verification variables, $\mathcal{I}\left(V_{t} ; \mathcal{Z}_{t}\right)$. The time derivative of the former can be written as

$$
\begin{aligned}
& \frac{d}{d t} \mathcal{I}\left(X_{t} ; \mathcal{Z}_{t}\right) \\
& \quad=\frac{1}{2} \operatorname{tr}\left\{\Sigma_{N}^{-1} C Q_{X} C^{\prime}\right\}-\frac{1}{2} \operatorname{tr}\left\{\Sigma_{W}\left(Q_{X}^{-1}-P_{X}^{-1}\right)\right\} .
\end{aligned}
$$

The first term depending on the observation represents the information supply and the second term depending on the process noise represents the information dissipation [14]. Similarly, the rate of $\mathcal{I}\left(V_{t} ; \mathcal{Z}_{t}\right)$ can be expressed as

$$
\begin{aligned}
& \frac{d}{d t} \mathcal{I}\left(V_{t} ; \mathcal{Z}_{t}\right) \\
& \quad=\frac{1}{2} \operatorname{tr}\left\{\Sigma_{N}^{-1} C Q_{X} M_{V}^{\prime}\left(M_{V} Q_{X} M_{V}\right)^{-1} M_{V} Q_{X} C^{\prime}\right\}+\beta(t),
\end{aligned}
$$

with some $\beta(t)$ that has no explicit dependency on $C$. It should be pointed out the sign of $\beta(t)$ is indefinite; $\mathcal{I}\left(V_{t} ; \mathcal{Z}_{t}\right)$ can increase without measurement and can decrease with measurement.

Proposition 4 The time derivative of the smoother form information gain at $t, \mathcal{J}_{V}^{S}(T, t) \triangleq \mathcal{J}_{0}(t)-\frac{1}{2} \operatorname{ldet}(I+$ $\left.Q_{X}(t) \Delta_{S}(t)\right)$, is written as:

$$
\frac{d}{d t} \mathcal{J}_{V}^{S}(T, t)=\frac{1}{2} \operatorname{tr}\left\{\Sigma_{N}^{-1} C(t) \Pi(t) C(t)^{\prime}\right\} \geq 0
$$

where $\Pi \triangleq Q_{X}\left(S_{X \mid V}-S_{X}\right)\left[I+Q_{X}\left(S_{X \mid V}-S_{X}\right)\right]^{-1} Q_{X}$. Since all the correlation through the process noise has already been captured in $S_{X \mid V}$, the mutual information rate is nonnegative regardless of the process noise. If one stops taking measurement at time $t$, the information reward stays constant. Thus, the mutual information rate for the smoother form can figure out the pure impact of sensing on the entropy reduction of the verification variables, while the rates for the filter forms depend on the process noise. 
Proof: The expression in (17) can be derived using $\frac{d}{d t} \operatorname{ldet} M_{1}=\operatorname{tr}\left\{M_{1}^{-1} \frac{d}{d t} M_{1}\right\}$ for $M_{1} \succ 0$, and the cyclic property of trace. Utilizing the matrix inversion lemma [23], $\Pi=Q_{X} \Delta_{S} Q_{X}-Q_{X} \Delta_{S}\left(Q_{X}^{-1}+\Delta_{S}\right)^{-1} \Delta_{S} Q_{X}=\Pi^{\prime}$. By the Wigner's theorem [24], $\Pi \succeq 0$; this leads to $\operatorname{tr}\left\{\Sigma_{N}^{-1} C \Pi C^{\prime}\right\} \geq 0$. See Proposition $\overline{6}$ in [22, Chapter 5] for details.

Remark 3 (Information Rate for Multiple Sensors) In case there are $m_{s}$ sensors constituting the overall observation matrix $C=\left[\begin{array}{lll}C_{1}^{\prime} & \ldots & C_{m_{s}}^{\prime}\end{array}\right]^{\prime}$, the mutual information rate can be written as

$$
\frac{d}{d t} \mathcal{J}_{V}^{S}(T, t)=\frac{1}{2} \sum_{i=1}^{m_{s}} \operatorname{tr}\left\{\Sigma_{N_{i}}^{-1} C_{i}\left(x_{i}, y_{i}\right) \Pi(t) C_{i}\left(x_{i}, y_{i}\right)^{\prime}\right\}
$$

where $(\mathbf{x}, \mathbf{y})$ represents the positions of the all the sensors, and $\Sigma_{N_{i}}$ is the $(i, i)$-th entry of $\Sigma_{N}$. In other words, the total rate of change of mutual information is the sum of rate of change of the mutual information of individual sensors.

Example 1 Fig. 2 compares the histories of the three on-thefly quantities: $\mathcal{J}_{V}^{S}(T, t)$ from the smoother form, $\mathcal{I}\left(X_{t} ; \mathcal{Z}_{t}\right)$ and $\mathcal{I}\left(V_{t} ; \mathcal{Z}_{t}\right)$ from the filter form. The following system matrices are used with $\tau=2$ and $T=5$ :

$A=\left[\begin{array}{cc}0.1 & 1 \\ -1 & -0.5\end{array}\right], \Sigma_{W}=\left[\begin{array}{cc}0.01 & 0 \\ 0 & 0.01\end{array}\right], P_{0}=\left[\begin{array}{cc}1 & 0.5 \\ 0.5 & 1\end{array}\right]$ $C=\left[\begin{array}{ll}0.5 & 0.5\end{array}\right], \Sigma_{N}=0.01, M_{V}=\left[\begin{array}{ll}0 & 1\end{array}\right]$.

Notice that $\mathcal{J}_{V}^{S}(T, t)$ at $t=\tau$ is the same as $\mathcal{I}\left(V_{t} ; \mathcal{Z}_{t}\right)$ at $t=T$, which confirms the equivalence of the filter form and the smoother form. For $\mathcal{J}_{V}^{S}(T, t)$, it is found that information increases in the presence of measurement and stays constant in the absence of measurement. In the history of $\mathcal{I}\left(X_{t} ; \mathcal{Z}_{t}\right)$, the information supply over $[0, \tau]$ increases the information while the information dissipation over $[\tau, T]$ decreases the information. The history of $\mathcal{I}\left(V_{t} ; \mathcal{Z}_{t}\right)$ is fluctuating; it can decrease with measurement and can increase without measurement, because information can be supplied/dissipated from/to the other state variables $X_{t} \backslash V_{t}$ via dynamics. In consequence, the filter form quantities, $\mathcal{I}\left(X_{t} ; \mathcal{Z}_{t}\right)$ and $\mathcal{I}\left(V_{t} ; \mathcal{Z}_{t}\right)$, cannot be a good indicator of the accumulated information $\mathcal{I}\left(V_{T} ; \mathcal{Z}_{t}\right)$; only the smoother form quantity $\mathcal{J}_{V}^{S}(T, t)$ represents the accumulated information.

\section{PATh REPRESENTATION}

The previous section suggests a formula to quantify the information gain for a continuous measurement history in a finite-dimensional linear system framework. This section shows how to relate the motion of a sensor in continuous space to a measurement history in the time domain, starting with the spatial interpolation method. This method describes the continuous field of the environmental variables in terms of a finite number of variables associated with the specified grid points. This work assumes that the environmental variables at location $\mathbf{r}$ can be represented as a linear combination of those at a finite number, $n_{G}$, of grid points $\mathbf{r}_{i}$ 's:

$$
\xi_{t}(\mathbf{r})=\sum_{i=1}^{n_{G}} \lambda_{i}\left(\mathbf{r}, \mathbf{r}_{i}\right) \xi_{t}\left(\mathbf{r}_{i}\right)
$$

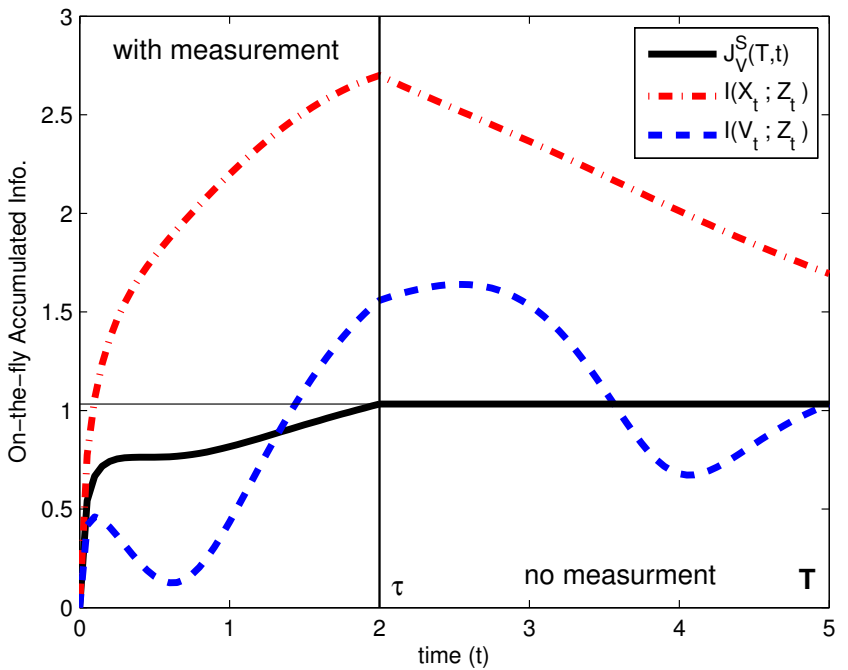

Fig. 2. On-the-fly information by a partial measurement path $\mathcal{Z}_{t}$ where $\xi_{t}(\cdot) \in \mathbb{R}^{n_{E}}$ represents the environmental variables associated with given location at time $t$, and the coefficient $\lambda_{i}$ is determined as a function of the location vectors $\mathbf{r}$ and $\mathbf{r}_{i}$. This paper considers time-invariant $\lambda_{i}$, although the results can be easily extended to time-varying cases. Many spatial interpolation techniques such as Kriging [5] and Gaussian processes regression (GPR) [6] provide expressions of the form in (18). In determining the coefficients for the linear combination, this paper specifically considers the zero-mean GPR method, which is equivalent to Simple Kriging, with squared exponential covariance function. The coefficients $\lambda_{i}\left(\mathbf{r}, \mathbf{r}_{i}\right)$ are expressed as $\lambda_{i}\left(\mathbf{r}, \mathbf{r}_{i}\right)=\sum_{j=1}^{n_{G}} \alpha_{i j} \rho\left(\mathbf{r}, \mathbf{r}_{j}\right)$ where $\rho\left(\mathbf{r}, \mathbf{r}_{j}\right)$ is defined as

$$
\rho\left(\mathbf{r}, \mathbf{r}_{j}\right) \triangleq \exp \left[-\frac{1}{2}\left(x-x_{j}\right)^{2} / l_{x}^{2}-\frac{1}{2}\left(y-y_{j}\right)^{2} / l_{y}^{2}\right]
$$

in the two-dimensional space, and $\alpha_{i j}$ is the $(i, j)$-th element of the matrix $\left[\rho\left(\mathbf{r}_{i}, \mathbf{r}_{j}\right)\right]^{-1}$. The parameters $l_{x}$ and $l_{y}$ represent the correlation length scales in each direction. For an isotropic environment, $l_{x}=l_{y}$ can be assumed, but for the atmospheric application considered in section VI, it is more reasonable to consider different length scales in each direction because the zonal and meridional variations of the weather are very different.

Under the assumption in (18), the environmental dynamics over the whole continuous space can be fully described by the dynamics of the finite number of variables at grid points. The state vector $X_{t} \in \mathbb{R}^{n}, n=n_{G} \times n_{E}$ is defined as $X_{t}=\left[\xi_{t}\left(\mathbf{r}_{i}\right)^{\prime} \cdots \xi_{t}\left(\mathbf{r}_{n_{G}}\right)^{\prime}\right]^{\prime}$, and this work considers linear dynamics for $X_{t}$ as in (1).

Consider a sensor located at $\mathbf{r}$ at time $t$ that receives measurement of $\xi_{t}(\mathbf{r})$. Since $\xi_{t}(\mathbf{r})$ is a linear combination of $\xi_{t}\left(\mathbf{r}_{i}\right)$ 's, the observation equation for this sensor can be expressed as $Z_{t}=C(t) X_{t}+N_{t}$ where $C(t) \in \mathbb{R}^{n_{E} \times n}$ is

$$
C(t)=\left[\begin{array}{lll}
\lambda_{1}\left(\mathbf{r}, \mathbf{r}_{1}\right) I_{n_{E}} & \cdots & \lambda_{n_{G}}\left(\mathbf{r}, \mathbf{r}_{n_{G}}\right) I_{n_{E}}
\end{array}\right] .
$$

For notational convenience, a single-sensor case will be considered in the later part of this paper on; extension to multiple sensors is straightforward.

If a sensor is continuously moving, its motion is fully described by the time history of the location vector $\mathbf{r}(t)$. 
Thus, the effect of the sensor's motion on the estimation system is through the evolution of the observation matrix $C(t)$ due to changes in $\lambda_{i}\left(\mathbf{r}, \mathbf{r}_{i}\right)^{\text {'s }}$ in time. Consider a sensor moving along a specified path $\mathbf{p}_{\tau}=\{\mathbf{r}(t): t \in[0, \tau]\}$ where $\mathbf{r}(t)$ is known for all $t \in[0, \tau]$. Then, the evolution of observation matrix $\mathcal{C}_{\tau}=\{C(t): t \in[0, \tau]\}$ can be derived by relating $C(t)$ and $\mathbf{r}(t)$. Then, the information gain associated with this path, denoted as $\mathcal{J}_{V}\left(T, \tau ; \mathbf{p}_{\tau}\right)$, can be computed by evaluating $Q_{X}\left(\tau ; \mathcal{C}_{\tau}\right)$, which is the final value of the Riccati equation corresponding to observation matrix history $\mathcal{C}_{\tau}$, while $\mathcal{J}_{0}$ and $\Delta_{S}$ have been computed in advance independently of $\mathbf{p}_{\tau}$.

To account for the limited mobility of the sensor, the path is, in general, represented as a set of equations of the location vector and its time derivatives: $f(\mathbf{r}(t), \dot{\mathbf{r}}(t), \ddot{\mathbf{r}}(t), \mathbf{u}(t))=\mathbf{0}$ where $\mathbf{u}$ is the control input for the sensor motion. For instance, a two-dimensional holonomic motion of a UAV sensor platform with constant speed $v$ can be written as

$$
\dot{x}(t)=v \cos \theta(t), \quad \dot{y}(t)=v \sin \theta(t)
$$

where $\theta(t)$ is the flight path angle, which is treated as a control input in this model.

\section{Path Planning Formulations}

\section{A. Optimal Path Planning}

The optimal motion planning determines the path $\mathbf{p}_{\tau}$, or equivalently the time history of the control input, that maximizes the smoother form information gain $\mathcal{J}_{V}^{S}(T, \tau)=$ $\mathcal{J}_{0}-\frac{1}{2} \operatorname{ldet}\left(I+Q_{X}\left(\tau ; \mathcal{C}_{\tau}\right) \Delta_{S}\right)$. The prior and posterior initial covariance $P_{0}$ and $P_{0 \mid V}$, respectively, are computed first; $\mathcal{J}_{0}$ and $S_{0}$ are computed using these information. Then, the optimization problem only involving the computation of $Q_{X}\left(\tau ; \mathcal{C}_{\tau}\right)$ is posed. This optimization problem is indeed a nonlinear optimal control problem (OCP) with a terminal cost functional. The control variables for this OCP are the controls for the sensor motion, e.g. $\theta(t)$ for two-dimensional holonomic motion, while there are two types of state variables: the vehicle position variables, $x$ and $y$, and the entries of the $Q_{X}(t)$ matrix. The optimal path planning problem for a two-dimensional holonomic mobile sensor is stated as

$$
\theta^{\star}(t) \in \arg \min _{\theta(t)} \operatorname{ldet}\left(I+Q_{X}\left(\tau ; \mathcal{C}_{\tau}\right) \Delta_{S}\right)
$$

subject to

$$
\begin{gathered}
\dot{Q}_{X}=A Q_{X}+Q_{X} A^{\prime}+\Sigma_{W}-Q_{X} C(x, y)^{\prime} \Sigma_{N}^{-1} C(x, y) Q_{X} \\
\dot{x}=v \cos \theta, \dot{y}=v \sin \theta \\
Q_{X}(0)=P_{0}, x(0)=x_{0}, y(0)=y_{0}
\end{gathered}
$$

where $C(x, y)$ is expressed as a function of $x$ and $y$ to emphasize that dependency in time is only through the evolution of $x$ and $y$. Regarding the size of this OCP, there is one control variable and the number of state variables is $n(n+1) / 2+2$. Constraints in the sensor's motion such as endpoint restriction, waypoint requirement, nonholonomic aspect of motion can be easily incorporated by modifying the vehicle's dynamics and by imposing additional constraints.
Also, multiple sensor problems can be dealt with by adding associated dynamic/kinematic constraints and by modifying the expression of the observation matrix.

\section{B. Information Potential Field and Real-Time Steering}

Optimal path planning gives a motion plan for maximum information reward, but, since it requires the solution of a nonlinear optimal control problem, it can be computationally expensive if $n$ is large due to a large state dimensions for the dynamic constraints. Thus, it is beneficial in practice to devise a computationally cheap feedback guidance law. One way to derive a real-time steering mechanism is to build some potential field and to move along the gradient of that field. The mutual information rate discussed in section III-C can be utilized to construct an information potential field. This type of information potential field extends a similar notion presented in [7], which derived the expression of $\frac{d}{d t}\left(\operatorname{ldet} Q_{X}^{-1}(t)\right)$ and neglected terms unrelated to the observation matrix to build a potential field. This section builds a potential field with the smoother form information rate in (17), which consists of a single term explicitly dependent on the observation matrix.

For the two-dimensional holonomic sensor motion in (20), the guidance law is presented as

$$
\theta_{G}(t)=\operatorname{atan} 2\left\{\frac{\partial}{\partial y}\left(\frac{d}{d t} \mathcal{J}_{V}^{S}(T, t)\right), \frac{\partial}{\partial x}\left(\frac{d}{d t} \mathcal{J}_{V}^{S}(T, t)\right)\right\}
$$

where $\frac{d}{d t} \mathcal{J}_{V}^{S}(T, t)$ is the smoother form mutual information rate, and atan2 denotes the four-quadrant arctangent.

Since the relationship between $C(x, y)$ and $(x, y)$ is known, the mutual information rate can be particularly written as a function of spatial coordinates: $\frac{d}{d t} \mathcal{J}_{V}^{S}(T, t)=$ $\frac{1}{2} \operatorname{tr}\left\{\Sigma_{N}^{-1} C(x, y ; t) \Pi(t) C(x, y ; t)^{\prime}\right\}$, and the gradient of the this expression can be evaluated accordingly. In case $C(x, y ; t) \in \mathbb{R}^{1 \times n}$, namely, there is only one environmental variable of interest, the spatial derivative can be written as

$$
\frac{\partial}{\partial p}\left(\frac{d}{d t} \mathcal{J}_{V}^{S}(T, t)\right)=\Sigma_{N}^{-1} C(x, y ; t) \Pi(t) \mathbf{d}(p), p=x, y
$$

where $\mathbf{d}(p)$ is an $n$-dimensional column vector whose $i$-th elements are $\mathbf{d}(p)_{i}=-l_{p}^{-2} \sum_{j} \alpha_{i j} \rho\left(\mathbf{r}, \mathbf{r}_{j}\right)\left(p-p_{j}\right), p=x, y$ In case $C$ is not a row vector, the relation in Remark 3 suggests that the mutual information rate and its gradient are nothing more than the sum of those with individual rows of the observation matrix.

\section{Numerical ExAMPLE}

This section deals with numerical implementation of the proposed path planning formulations, to confirm that the linear estimation theoretic formula together with the path representation technique realizes the continuous measurement path for mobile sensors.

\section{A. Model and Scenario}

A simplified weather forecast problem is considered for numerical verification. This paper employs the same twodimensional Lorenz-2003 model as used in [20]. The system 
equations are

$$
\begin{aligned}
\dot{\xi}_{i j} & =-\xi_{i j}-\zeta_{i-4, j} \zeta_{i-2, j}+\frac{1}{3} \sum_{k \in[-1,1]} \zeta_{i-2+k, j} \xi_{i+2+k, j} \\
& -\mu \eta_{i, j-4} \eta_{i, j-2}+\frac{\mu}{3} \sum_{k \in[-1,1]} \eta_{i, j-2+k} \xi_{i, j+2+k}+F
\end{aligned}
$$

where $\zeta_{i j} \triangleq \frac{1}{3} \sum_{k \in[-1,1]} \xi_{i+k, j}, \quad \eta_{i j} \triangleq \frac{1}{3} \sum_{k \in[-1,1]} \xi_{i, j+k}$ for $(i, j) \in\left[1, L_{i}\right] \times\left[1, L_{j}\right]$. The subscript $i$ denotes the westto-eastern grid index, while $j$ denotes the south-to-north grid index. The boundary conditions of $y_{i+L_{i}, j}=y_{i-L_{i}, j}=y_{i, j}$ and $y_{i, 0}=y_{i,-1}=3, y_{i, L_{j}+1}=0$ in advection terms, are applied to model the mid-latitude area as an annulus. The parameter values are $L_{i}=72, L_{j}=17, \mu=0.66$ and $F=$ 8. The size of $1 \times 1$ grid corresponds to $347 \mathrm{~km} \times 347$ $\mathrm{km}$ in real-life distance, and 0.05 time units in this model is equivalent to 6 hours of real-life time. The overall system is tracked by a nonlinear estimation scheme, specifically a ensemble square-root filter (EnSRF) [25] data assimilation scheme, with incorporating a fixed observation network of size 186.

The path planning problem is posed for the linearized model for the local region defined by the indices $(i, j) \in$ $[47,50] \times[12,14]$. A linear invariant model is obtained by deriving the Jacobian matrix of the dynamics around the nonlinear estimate for $\xi_{i j}$ 's at the grid points in the local region. Thus, the state vector $X_{t} \in \mathbb{R}^{n}, n=12$ represents the perturbation of the $\xi_{i j}$ 's from the ensemble mean. In this linear model, the dependence of the local dynamics on the evolution of the outside dynamics is ignored in deriving the Jacobian matrix (or $A$ matrix). Instead, this effect is incorporated in the process noise term, i.e. the states on the boundary of the local region, which may be effected by outside dynamics more substantially, are assumed to be subject to larger process noise. The correlation length scale parameters for spatial interpolation are chosen as $l_{x}=$ $1, l_{y}=0.7$ grids.

The goal is to design a 6-hr flight path ( $\tau=6 \mathrm{hrs}$ ) for a single UAV sensor platform to improve the forecast over the right-most three grid points in the local region in 72 hrs $(T=72 \mathrm{hrs})$. The motion of the UAV is described as 2-D holonomic motion and it flies at constant speed $v=1 / 3 \mathrm{grid} / \mathrm{hr}$, equivalently, $116 \mathrm{~km} / \mathrm{hr}$. The initial location of the UAV is $(49,13)$. The prior and posterior initial covariance matrices, $P_{0}$ and $P_{0 \mid V}$ are provided by the EnSRF data assimilation scheme, where $P_{0 \mid V}$ is computed by the ensemble augmentation method [21].

\section{B. Results}

Two proposed path planning methods, optimal path planning and the gradient-based real-time steering, are compared with the myopic versions of them. The myopic path planning takes into account $\mathcal{I}\left(X_{\tau} ; \mathcal{Z}_{\tau}\right)$ instead of $\mathcal{I}\left(V_{T} ; \mathcal{Z}_{\tau}\right)$, the underlying hypothesis being that uncertainty reduction in the current estimate would lead to uncertainty reduction in the future forecast. More specifically, the optimal myopic solution minimizes ldet $Q_{X}(\tau)$, and the myopic real-time steering utilizes the filter form information rate in (16) to construct an information potential field.
TABLE I

INFORMATION GAINS FOR DIFFERENT STRATEGIES

\begin{tabular}{c|c|c|c|c|c} 
Opt & RT & Opt. Myo. & Myo. RT. & Best SL & Worst SL \\
\hline \hline 0.69 & 0.62 & 0.20 & 0.14 & 0.43 & 0.14
\end{tabular}

Each of the two optimal control problem is formulated as a nonlinear program (NLP) by parameterizing the control history as a piecewise linear functions with 12 segments. TOMLAB/SNOPT v6.0 [26] is used to solve NLPs; realtime steering solutions, and various straight-line solutions are used as initial guess for the optimization. Solutions of NLPs are obtained within two minutes and satisfy first-order optimality criteria. The best and the worst straight-line paths are also considered for comparison. The best straight-line strategy solves an NLP to find the constant flight path angle $\theta_{0}$ that maximizes the smoother form information gain.

Table I represents the information gains $\mathcal{J}_{V}^{S}(T, \tau)$ for the considered methods. It is noted that the information gains for the two myopic solutions are highly suboptimal - almost close to the worst straight-line. Fig. 3 depicts the UAV trajectories for different strategies overlaid with the information field at the initial time. Trajectories for the myopic decisions (plus the worst straight) head northward, while others head southward where the information potential field indicates a high information peak. It turns out that the myopic information field based on the filter form information rate in (16) suggests that there is an information peak in the north-western part of the grid region; this is why the two myopic decisions head northward [22]. Fig. 4 represents the accumulated information gains, $\mathcal{I}\left(V_{T} ; \mathcal{Z}_{t}\right), t<\tau$, for different strategies. The optimal trajectory is not a virtually attractive route in a short-term view (up to $2 \mathrm{hrs}$ ), but a large amount of information is gathered in the later part of the optimal trajectory. The optimal strategy sometimes sacrifices immediate reward in order to lead the sensor to better site at a later time. Fig. 5 shows the optimal trajectory snapshot every hour overlaid on the information potential field at each time. Note that variation in the potential field is solely due to the measurement, since the smoother form does not have information dissipation.

\section{CONCLUSIONS}

A methodology for continuous motion planning of sensors for information forecast was presented. The key contribution of this work is to provide the framework of quantifying information by a continuous measurement path. The smoother form formula of the information gain that reduces the timescale of the decision space enhanced computational efficiency and enabled on-the-fly evaluation of the accumulated information. An optimal path planning formulation and a real-time steering law were presented with employing spatial interpolation for path representation. A numerical example for the simplified weather forecast verified the applicability of the proposed theoretical framework. Future work will extend the proposed method to problems in other domains.

\section{ACKNOWLEDGMENT}

This work is funded by NSF CNS-0540331 as part of the DDDAS program with Dr. Frederica Darema as the overall 


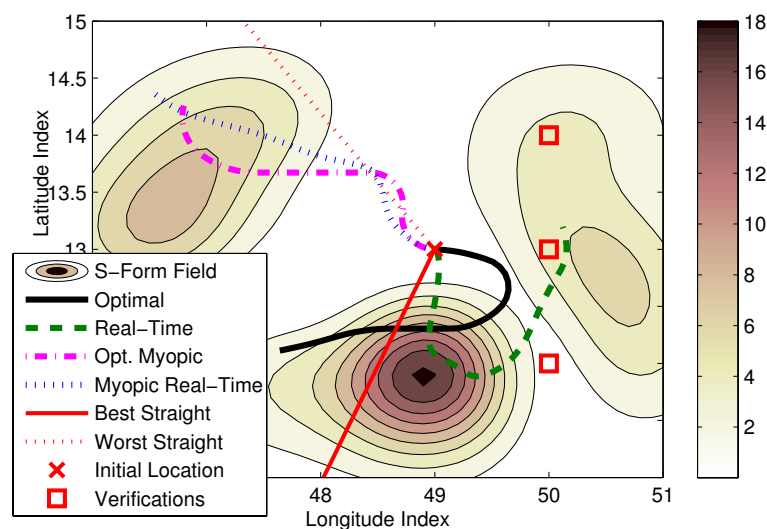

Fig. 3. Sensor trajectories for the different strategies: myopic solutions fail to move toward the information-rich southern region.

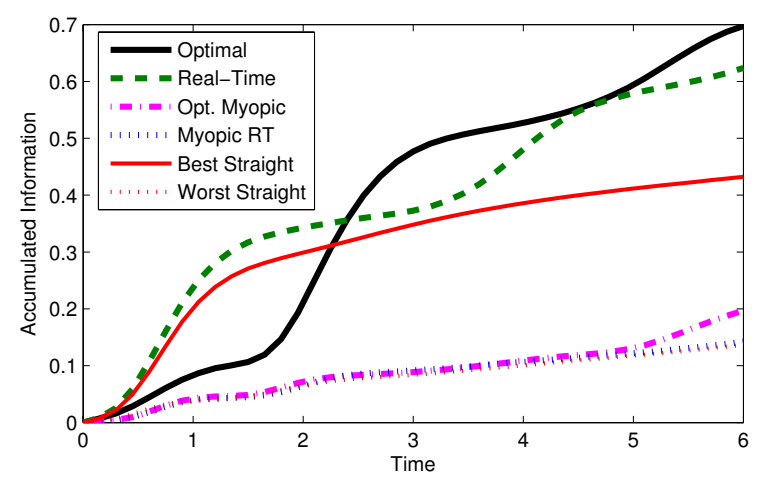

Fig. 4. Accumulated information for the four strategies: the optimal solution sacrifices immediate reward to gather more information at a later time.

program manager. The authors thank Luca Bertuccelli for insightful discussions.

\section{REFERENCES}

[1] B. Grocholsky, A. Makarenko, and H. Durrant-Whyte, "InformationTheoretic Coordinated Control of Multiple Sensor Platforms," IEEE Int. Conf. on Robotics and Automation, Taipei, Taiwan, Sep. 2003.

[2] G.M. Hoffmann, S.L. Waslander, and C.J. Tomlin, "Mutual Information Methods with Particle Filters for Mobile Sensor Network Control," IEEE Conf. on Decision and Control, Dec. 2006.

[3] M.S. Arulampalam, B. Ristic, N. Gordon, and T. Mansell, "BearingsOnly Tracking of Manoeuvring Targets using Particle Filters," EURASIP Journal of Applied Signal Processing, Vol. 15, pp. 23512365, 2004.

[4] S. Martinez and F. Bullo, "Optimal Sensor Placement and Motion Coordination for Target Tracking," Automatica, Vol. 42, pp. 661-668, 2006.

[5] N. Cressie "The Origins of Kriging," Mathematical Geology, Vol. 22, No. 3, 1990.

[6] C.K.I. Willams and C.E. Rasmussen, "Gaussian Processes for Regression," Advances in Neural Information Processing Systems 8, MIT Press, 1996.

[7] B. Grocholsky, "Information-Theoretic Control of Multiple Sensor Platforms," PhD Dissertation, University of Sydney, 2002.

[8] T.E. Duncan, "On the Calculation of Mutual Information," SIAM Journal on Applied Mathematics, Vol. 19, No. 1, July 1970.

[9] T.T. Kadota, M. Zakai, and J. Ziv, "Mutual Information of the White Gaussian Channel With and Without Feedback," IEEE Trans. on Information Theory, Vol. IT-17, No. 4, 1971.

[10] D. Guo, S. Shamai (Shitz), and S. Verdú, "Mutual Information and Minimum Mean-Square Error in Gaussian Channels," IEEE Trans. on Information Theory, Vol.51, No.4, 2005.

[11] T.E. Duncan and B. Pasik-Duncan, "Estimation and Mutual Information," The 46th IEEE Conf. on Decision and Control, New Orleans, LA, Dec. 2007.
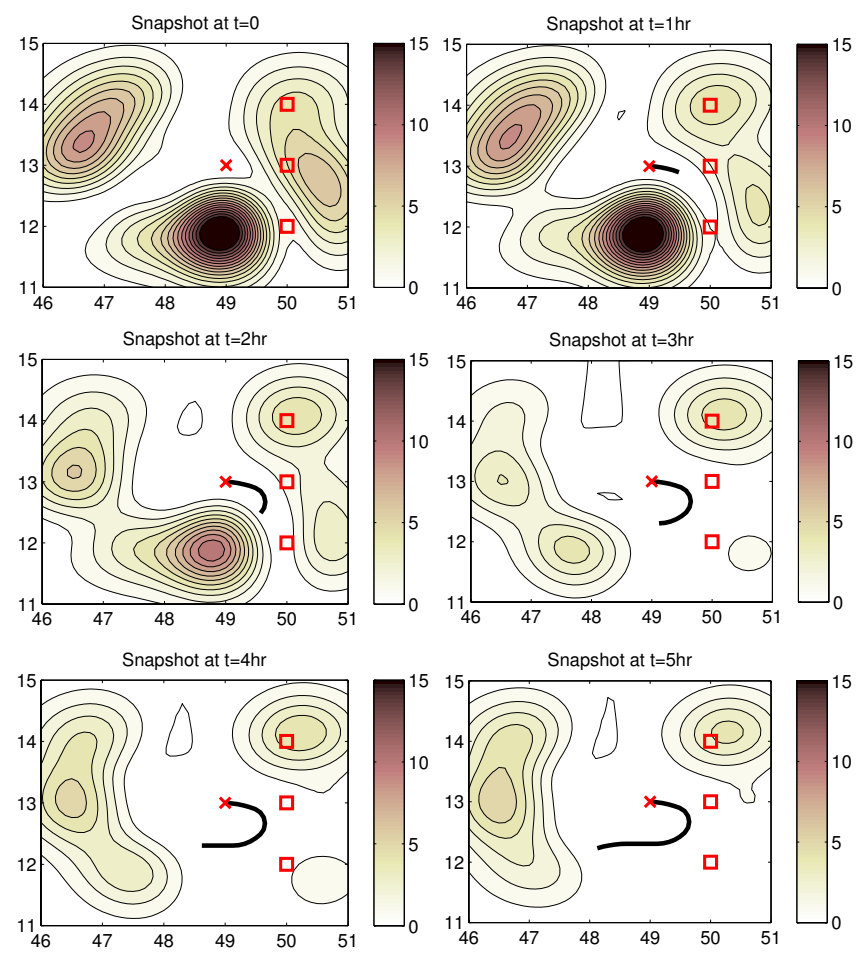

Fig. 5. Snapshots of the optimal trajectory and the information potential field: a large piece of information located in the south is gathered between $2 \mathrm{hr}$ and $3 \mathrm{hr}$.

[12] Y. Tomita, S. Omatu, and T. Soeda, "An Application of the Information Theory to Filtering Problems," Information Sciences, Vol. 11, pp. 1327. 1976.

[13] E. Mayer-Wolf and M. Zakai, "On a Formula Relating the Shannon Information to the Fisher Information for the Filtering Problem," Lecture Notes in Control and Information Sciences 61, pp. 164-171, 1984.

[14] S.K. Mitter and N.J. Newton, "Information and Entropy Flow in the Kalman-Bucy Filter," Journal of Statistical Physics, Vol.118, No.112, Jan. 2005.

[15] N.J. Newton, "Dual Kalman-Bucy Filters and Interactive Entropy Production," SIAM Journal on Control and Optimization, Vol. 46, No. 5, pp. 1637-1663, 2007.

[16] N.J. Newton, "Dual Nonlinear Filters and Entropy Production," SIAM J. on Control and Optimization, Vol. 45, No. 3, pp. 998-1016, 2006.

[17] J.E. Wall Jr., A.S. Willsky, and N.R. Sandell Jr., "On the Fixed-Interval Smoothing problem," Stochastics, Vol. 5, No.1\&2, pp. 1-41, 1981.

[18] T.N. Palmer, R. Gelaro, J. Barkmeijer, and R. Buizza, "Singular Vectors, Metrics, and Adaptive Observations," Journal of the Atmospheric Sciences, Vol 55, No.4, pp.633-653, 1998.

[19] S.J. Majumdar, C.H. Bishop, B.J. Etherton, and Z. Toth, "Adaptive Sampling with the Ensemble Transform Kalman Filter: Part II Field Programming Implementation," Monthly Weather Review, Vol.130, No.3, pp.1356-1369, 2002.

[20] H.-L. Choi and J.P. How, "A Multi-UAV Targeting Algorithm for Ensemble Forecast Improvement," AIAA Guidance, Navigation, and Control Conference, Aug. 2007.

[21] H.-L. Choi and J.P. How, "Efficient Targeting of Large-Scale Sensor Networks," IEEE Trans. on Signal Processing, submitted, May 2008.

[22] H.-L. Choi, "Adaptive Sampling and Forecasting With Mobile Sensor Networks," PhD Thesis, Dept. Aeronautics and Astronautics, MIT, Sept. 2008, http://web.mit.edu/hanlimc/Public/thesis.pdf.

[23] R.A. Horn and C.R. Johnson, Matrix Analysis, Cambridge University Press, 1985.

[24] R. Subramanian and K.V. Bhagwat, "On a Theorem of Wigner on Products of Positive Matrices," Proceedings Mathematical Sciences, Vol. 88 A, Part 3, No. 1, pp.31-34, 1979.

[25] J.S. Whitaker and H.M. Hamill, " Ensemble Data Assimilation without Perturbed Observations," Monthly Weather Review, Vol. 130, pp. 19131924, 2002

[26] http://tomopt.com/tomlab/products/snopt/ 